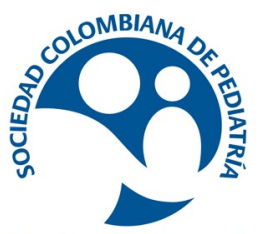

Revisión Sistemática

\title{
Validez diagnóstica de las pruebas clínicas para el diagnóstico diferencial de Dengue en población pediátrica.
}

\author{
Mayra Mercedes Rubiano Godoy y Beatriz Stella Jimenez Cendales. \\ a Universidad CES de Medellín, Facultad de Medicina y Universidad Nuestra Señora del Rosario, Facultad de Ciencias de la Salud, Bogotá D.C. \\ Posgrado Maestría en Epidemiología. Colombia.
}

\section{INFORMACIÓN DEL ARTÍCULO}

Historia del artículo:

Recibido el 29 de octubre 2019

Aceptado el 15 de febrero 2021

Palabras clave:

Infecciones por Arbovirus

síntomas y signos

sensibilidad y especificidad

técnicas y procedimientos diagnósticos pediatría

Keywords:

Arbovirus infections

signs and symptons

sensitivity and specificity

diagnostic techniques and

procedures

pediatrics

\section{R E S U M E N}

\begin{abstract}
Antecedentes: El dengue es un problema creciente de salud pública, por lo que ha surgido como reto, la implementación de un diagnóstico diferencial basado en síntomas y signos clínicos y paraclínicos, especialmente en la población pediátrica más afectada según la evidencia epidemiológica. Objetivo: Determinar la validez diagnóstica de las pruebas clínicas para el diagnóstico diferencial del dengue en población pediátrica. Metodología: Se realizó una revisión sistemática de literatura incluyendo estudios primarios de pruebas diagnósticas de dengue, en población pediátrica. Se efectuó la búsqueda en las bases de datos MEDLINEPUBMED, EMBASE, COCHRANE y LILACS. La calidad se evaluó con la herramienta QUADAS-2. Registro protocolo PROSPERO: CRD42019125201. Resultados: Se incluyeron 3 estudios de Taiwan, Puerto Rico y Cambodia, que cumplieron los criterios de inclusión, aunque con gran heterogeneidad metodológica. La prueba índice incluyó una variedad de combinaciones de síntomas y signos asociados a la fiebre para diagnosticar dengue. El rash presentó sensibilidad entre $27.8 \%$ y $76.1 \%$ y especificidad entre $55.4 \%$ y $95.5 \%$ con VPP entre $76.7 \%$ y $86.1 \%$ que aumentó al $100 \%$ en combinaciones de síntomas y signos. La combinación leucopenia, trombocitopenia y elevación de transaminasas alcanzó VPP del 100 \%. Conclusión: La combinación de pruebas confirmatorias afecta las características operativas de cada patrón de oro configurado, impidiendo su comparación. La combinación de [eritema + prurito + petequias] indica la posibilidad de construir un algoritmo diagnostico. La evidencia insuficiente encontrada sugiere la necesidad de desarrollar estudios prospectivos estandarizados de evaluación clínica basada en validez diagnóstica de los síntomas y signos para el diagnóstico diferencial del dengue.
\end{abstract}

\section{Accuracy test of clinical trials for the differential diagnosis of dengue in} the pediatric population.

A B S T R A C T

Background: Dengue is a public health problem with a growing incidence, whereby it has emerged as a dare the implementation of a differential diagnosis based on clinical symptoms

\footnotetext{
*Autor para correspondencia. Mayra Mercedes Rubiano Godoy

Correo electrónico: mayra.rubiano@urosario.edu.co
}

Como Citar: Rubiano Godoy MM, Jimenez Cendales BS., Validez diagnóstica de pruebas clínicas para el diagnóstico diferencial de Dengue. Pediatr. 2020;53(4):122-130. 
and signs and clinical laboratory test, for the most affected: pediatric population. Objective: To determine the diagnostic validity of clinical tests for the differential diagnosis of dengue in the pediatric population. Methods: A systematic review of the literature was carried out, including primary studies of dengue diagnostic tests, in the pediatric population. Searches were conducted in MEDLINE-PUBMED, EMBASE, COCHRANE, and LILACS databases. The quality of the studies was evaluated with the QUADAS-2 tool. Register PROSPERO: CRD42019125201. Results: Three studies from Taiwan, Puerto Rico, and Cambodia that met the inclusion criteria were included, although with significant methodological heterogeneity. The index test consists of various combinations of symptoms and signs associated with fever to diagnose dengue. Rash had sensitivity between $27.8 \%$ to $76.1 \%$ and specificity between 55.4 to $95.5 \%$, with a PPV between 76.7 and $86.1 \%$ that increased to $100 \%$ in various combinations of symptoms and signs. The combination leukopenia, thrombocytopenia, and transaminase elevation had a PPV of $100 \%$. Conclusion: The varied combination of confirmatory tests affects each configured gold standard's operational characteristics, thus preventing their comparison. The combination of [rash + pruritus + petechiae] suggests the possibility of building a diagnostic algorithm. Insufficient evidence indicates the need to develop standardized prospective clinical evaluation studies based on diagnostic validity of symptoms and signs for differential diagnosis of dengue.

\section{Introducción}

Las infecciones arbovirales, como el dengue, son un problema de interés en salud pública a nivel mundial, especialmente en regiones tropicales, en las cuales se favorece la circulación del vector que las transmite, con la consecuente propagación y diseminación de los virus en los entornos urbanos (1,2). Entre el 2000 y 2015 se notificaron 15.9 millones de casos de personas infectadas y 7.000 muertes atribuidas etiológicamente en América Latina (2,3). Dichas cifras tienden a aumentar a pesar de la implementación de estrategias para el manejo de vectores, en especial en países como Brasil, Colombia y México que registran el $85 \%$ de los casos del continente (4). En el 2017, la incidencia mundial promedio del dengue mostró una tendencia especialmente marcada en la población infantil y de adolescentes, con preocupante letalidad $(5,6)$.

La infección causada por el virus del dengue constituye un reto para el diagnóstico clínico, la confirmación por laboratorio y la vigilancia epidemiológica en la población pediátrica $(6,7)$, debido principalmente a que comparte un espectro muy amplio de manifestaciones clínicas con otras infecciones de virus trasmitidos por vectores como el Chikunguña y el Zika, entre las cuales están la fiebre, las artralgias y mialgias, la cefalea, el rash maculopapular, la conjuntivitis, la linfadenopatía, la leucopenia, la trombocitopenia, la astenia y la adinamia. Sin embargo, las características clínicas de cada una de estas manifestaciones pueden cambiar por muchas razones, resaltando la necesidad de una minuciosa anamnesis por parte del profesional médico, con la cual tenga la capacidad de obtener un diagnóstico diferencial acertado, especialmente en la población pediátrica $(2,6,7)$ y seleccionar la conducta pertinente, según su sospecha clínica, para garantizar la acertada interpretación de los resultados (2,6,8-12).

Se sugiere establecer un diagnóstico clínico con base en la semiología acuciosa y el contexto epidemiológico, que oriente la conducta médica y la necesidad de la confirmación paraclínica directa o indirecta en los casos del dengue, según el tiempo de evolución de la enfermedad $(2,4,6,7)$.
Para el diagnóstico del dengue se utilizan principalmente tres tipos de pruebas: 1) El aislamiento viral e identificación de la proteína no estructural NS1 (Del Inglés: Nonstructural protein 1), 2) La Reacción en Cadena de la Polimerasa en Tiempo Real (RT-PCR) y 3) La serología. Técnicas a través de las cuales se busca confirmar la etiología responsable de la enfermedad sospechada. Sin embargo, en los lugares endemoepidémicos para dengue, es poco frecuente aplicar las tres o cualquiera de las pruebas, en todas las personas con sospecha clínica de esta infección, pues la aplicación de dichas pruebas exige recursos económicos, infraestructurales, de insumos (preservación de muestras, reactivos) y humanos (personal especializado), no disponibles en la mayor parte de las regiones tropicales endémicas $(13,14)$.

De la misma manera, los tres tipos de pruebas tienen limitaciones propias de la técnica. En el caso del aislamiento viral, la muestra debe ser recolectada dentro de los seis primeros días de iniciados los síntomas de dengue. De lo contrario, no se logrará detectar viremia. Con respecto a la RT-PCR (Del inglés real time polymerase chain reaction), se requiere de la experiencia del evaluador para obtener resultados confiables. Finalmente, en la serología existen reacciones cruzadas entre anticuerpos de los arbovirus a los que han estado previamente expuestas las personas, con lo que se generan resultados falsos (15)dengue y Zika (flavivirus).

Por lo anterior, el objetivo de la presente revisión es determinar la validez diagnóstica de las pruebas clínicas para el diagnóstico diferencial de la infección por el virus del dengue en la población pediátrica, describiendo las características de los participantes en los estudios incluidos en la revisión, identificando los métodos utilizados para el diagnóstico clínico de la infección, especificando las características operativas de las pruebas diagnósticas clínicas y paraclínicas frente al patrón de referencia reportado y resumiendo los criterios de diagnóstico diferencial utilizados para la identificación clínica de la infección por el virus del dengue. El uso de herramientas de predicción que incrementen la probabilidad diagnóstica y estandarización del enfoque clínico epidemiológico diferencial conlleva el mejoramiento de resultados en salud en la población pediátrica. 


\section{Metodología}

Se realizó una revisión sistemática de la literatura, la cual fue registrada en PROSPERO (De las siglas en inglés de: International Prospective Register of Systematic Reviews) Registro No. CRD42019125201. Se incluyeron estudios primarios de pruebas de validez diagnóstica para dengue publicados a partir de noviembre 2009 (momento en el cual la OMS unificó los términos de diagnóstico de la infección), sin restricción de lenguaje, en los cuales fue posible extraer datos en términos de sensibilidad, especificidad, valores predictivos positivos y negativos del procedimiento de diagnóstico utilizado, en población menor de 18 años. La prueba índice la constituyen los síntomas o signos clínicos, individuales o un grupo de ellos, acompañados o no de prueba generales de laboratorio clínico. El patrón de referencia lo componen las pruebas de laboratorio confirmatorias, tales como técnicas de aislamiento viral y RT-PCR, combinados o no con pruebas serológicas para la detección del anticuerpo antidengue (IgM específicos), de reconocimiento de NS1 e inmunohistoquímicas en especímenes biológicos o muestras de patología. Fueron excluídos estudios sin confirmación mediante patrón de referencia, de población en estado de inmunosupresión, reporte de casos y series de casos.

La estrategia de búsqueda utilizando operadores booleanos

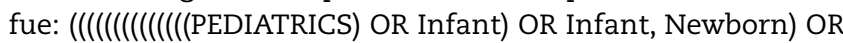
Child) OR Child, Preschool) OR Adolescent) OR "Pediatrics"[Mesh]) OR "Infant"[Mesh]) OR "Infant, Newborn"[Mesh]) OR “Child”[Mesh]) OR "Child, Preschool"[Mesh]) OR “Adolescent”[Mesh])) AND ( (()(()(()(()(((Signs and symptoms)) OR Clinical manifestations) OR Clinical presentation) OR Clinical features) OR Clinical form) OR Clinical representation) OR Clinical expression) OR Clinical commitment) OR Clinical commitment) OR Clinical status) OR Clinical findings) OR Clinical diagnosis) OR Clinical alterations) OR Clinical condition) OR ("Signs and Symptoms"[Mesh])) OR ("Pathological Conditions, Signs and Symptoms"[Mesh]))) AND

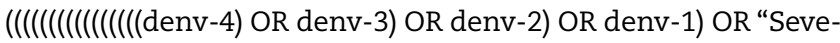
re Dengue/diagnosis"[Mesh]) OR "Severe Dengue"[Mesh]) OR "Dengue/diagnosis"[Mesh]) OR “Dengue"[Mesh]) OR Dengue Virus) OR Dengue infection) OR Dengue fever) OR Severe dengue) OR Dengue with warning signs) OR Dengue without warning signs) OR Dengue) OR Dengue disease) AND ((“2009/01/01”[PDat] : “2019/12/31”[PDat]) AND Humans[Mesh]).

La búsqueda se realizó en las bases de datos MEDLINE a través de Pubmed, Cochrane Library, EMBASE (De las siglas en inglés de: Excerpta Medica dataBASE), LILACS (De las siglas: Literatura latinoamericana y del Caribe en Ciencias de la salud), CINAHL (De las siglas en Ingles de: The Cumulative Index to Nursing and Allied Health Literature), así como literatura gris, repositorios universitarios, memorias de simposios, actas de congresos, informes de investigaciones y proyectos nacionales e internacionales detallados en el protocolo de esta revisión, listas de referencia y búsqueda manual en publicaciones y referencias bibliográficas encontradas hasta febrero 08 de 2019 , siguiendo las etapas de identificación, cribado, elegibilidad e inclusión expuestas en la guía PRISMA (De las siglas en inglés: Preferred Reporting Items for Systematic Reviews and Meta-Analyses) (16).
Dos revisores independientes seleccionaron los estudios, un tercer revisor actuó como árbitro para resolver las diferencias de criterio.

La calidad de los estudios fue evaluada utilizando la herramienta QUADAS-2 (De las siglas en inglés de: Quality Assessment of Diagnostic Accuracy Studies) (17). Acorde con el protocolo, se procuró controlar y disminuir posibles sesgos de selección e información, incluyendo sesgos de publicación y del investigador.

Se realizó un análisis descriptivo acerca de las características de los estudios incluidos, a partir de los cuales se construyó una tabla de $2 \times 2$ obtenendo una clasificación cruzada binaria entre las pruebas clínicas y los marcadores de laboratorios pertinentes.

\section{Resultados}

A partir de la estrategia de búsqueda y luego de eliminar las referencias duplicadas, se obtuvieron 1816 referencias. El proceso de selección se resume en el gráfico 1.

Respecto al serotipo del virus del dengue, éste fue identificado en cada uno de los estudios, pero, Cavailler y cols. en el 2016 no discriminaron cada uno de los signos y síntomas evaluados según el serotipo implicado. Además, Chen y cols. en el 2018 y Ramos y cols. en el 2009, consideraron el diagnóstico de dengue en general, mientras que Cavailler y su grupo se enfocó solamente en el diagnóstico de dengue con signos de alarma. Así, la prueba índice varió entre los estudios seleccionados.

En los 3 estudios se consideró la fiebre, pero de acuerdo con Cavailler en el 2016 esta condición se definió como temperatura mayor a $37.5^{\circ} \mathrm{C}$. Además de la fiebre, se tuvieron en cuenta cuadros clínicos diversos. Según Chen, se consideraron algunos síntomas y signos individualmente en el análisis, tales como eritema, prurito, petequias, cefalea, tos, entre otros y las combinaciones clínicas de: [eritema + cefalea]; [petequias + cefalea]; [eritema + prurito + petequias]. De igual manera, Ramos y cols. en el 2009, exponen signos y síntomas individuales como cefalea, mialgia, eritema y mialgia, entre otros, o ciertas combinaciones en relación con el eritema, midiendo las diferencias en diverso número de sujetos con confirmación diagnóstica (Tablas 1 y 2).

En general se considera que los 3 estudios incluidos (Chen, 2018; Ramos, 2009 y Cavailler, 2016) son de adecuada calidad metodológica, aplicando los criterios de evaluación de QUADAS-2, como se observa en los gráficos 2 y 3. Sin embargo, exceptuó pacientes menores de 5 y mayores de 15 años. Además, Ramos y Cavailler no describen de manera clara el intervalo de tiempo entre la aplicación o evaluación de las pruebas de índice y el estándar de referencia. De otra parte, tanto en el estudio de Ramos como en el de Cavailler, no es claro que a todos los pacientes se les haya tomado la misma prueba de referencia. En el caso del estudio de Chen, se sabe las pruebas de referencia utilizadas, pero se desconoce el número de pacientes a los cuales les aplicaron cada una de ellas.

No se alcanzó el número suficiente de estudios incluidos en la revisión sistemática y la heterogeneidad metodológica de los incluíudos imposibilitaron la realización de un metaanálisis, como se observa en la tabla 2. 


\section{Discusión}

La identificación de la validez diagnóstica de las pruebas clínicas para el diagnóstico diferencial de la infección por el virus del dengue en la población pediátrica soporta la magnitud de su impacto en el número de niños y adolescentes que padecen la enfermedad. Bhattacharya y cols. en 2013 (21) proponen que cerca del $95 \%$ de las infecciones causadas por el virus del dengue se presentan en personas menores de 15 años de edad, lo que concuerda con los resultados de los estudios incluidos en esta revisión, en los cuales la mediana de edad oscila entre los 9 y 10 años de edad.

En revisiones sistemáticas previas como la realizada por Guo y cols. en 2017 (22), se postula que la población pediatrica infectada por el virus del dengue a nivel mundial se caracteriza por proceder de condiciones sociales y económicas relativamente bajas. Sin embargo, a pesar de que no se describen dichas características entre los participantes incluidos en los estudios analizados en esta revisión, es claro que las condiciones socioeconómicas de Taiwán, Puerto Rico y Camboya varían de un país a otro.
El elemento común más evidente que comparten estos países es su posicionamiento geográfico con respecto al trópico ecuatorial, coincidiendo con autores como Messina y cols. en el 2015 (23) quienes sugieren, por medio de un mapa global de idoneidad ambiental para presencia del dengue, que las intervenciones de prevención se enfoquen en dicho aspecto, debido a la alta densidad de población y el ambiente ideal para el mantenimiento del vector, que facilita la trasmisión del virus.

Acerca de la prueba índice, el eritema, el dolor retro ocular, la mialgia y las náuseas o vómito fueron los únicos signos y síntomas comunes en la población incluida en los tres estudios que integran la presente revisión, a pesar de su heterogeneidad y de la metodología empleada en cada uno de estos estudios, sugiriendo que cada uno de estos signos y síntomas, a pesar de estar presentes, de manera aislada no presentan relevancia predictiva para el diagnostico del dengue.

Pese a tener claro que la edad no es un determinante exclusivo para la presentación clínica del dengue, aquellos análisis de prueba diagnósticas que utilicen como prueba índice la combinación de signos y síntomas que acompañan a la fiebre, como [eritema + prurito + petequias] (18), abren la posibilidad de construir un algoritmo diagnostico en niños en quien se

Gráfico 1. Diagrama PRISMA para Revisiones Sistemáticas de la Literatura

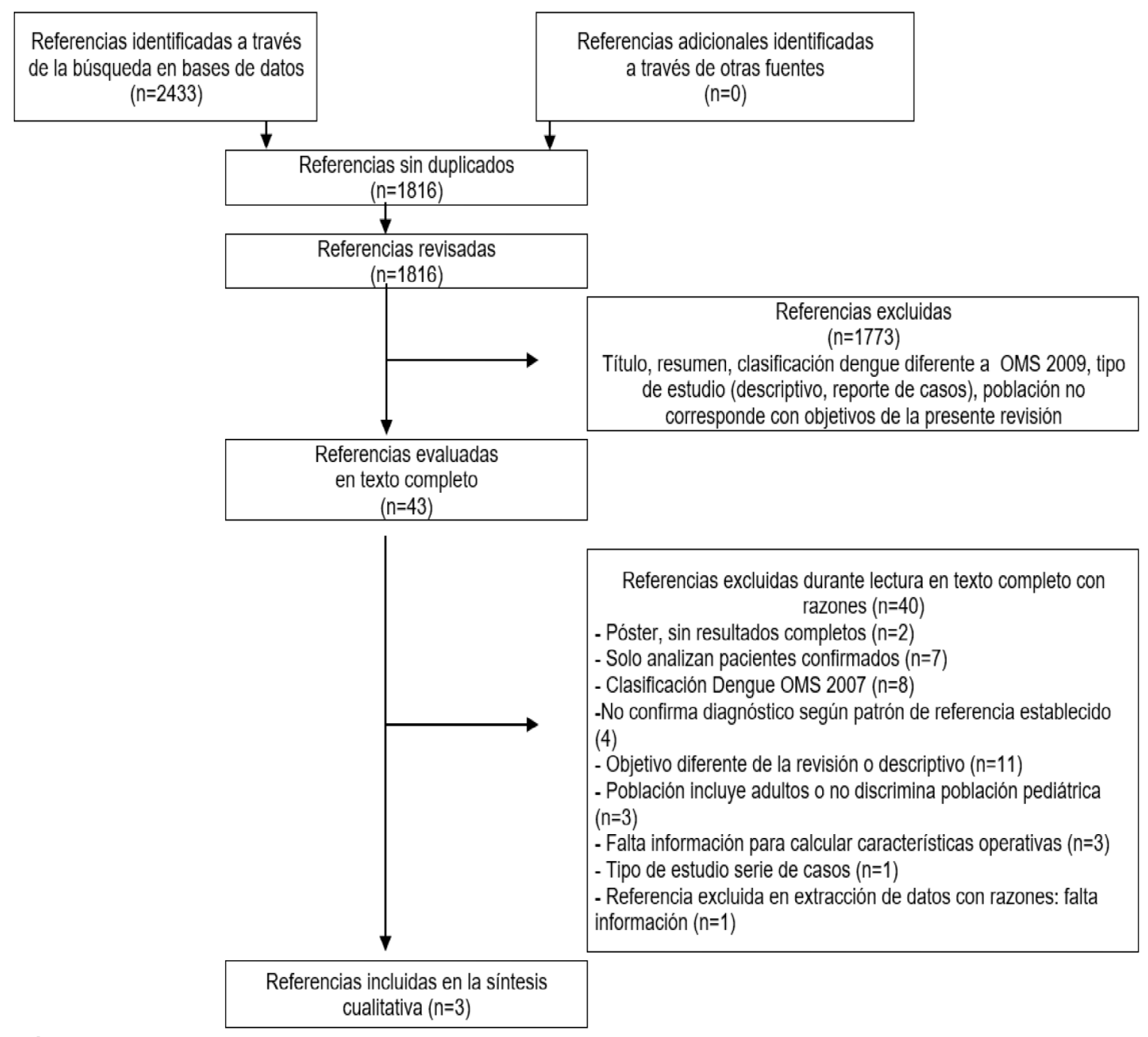


Tabla 1. Características de los artículos incluidos

\begin{tabular}{|c|c|c|c|}
\hline \multirow{2}{*}{ Características } & \multicolumn{3}{|c|}{ Identificación del artículo } \\
\hline & Chen 2018 & Ramos 2009 & Cavailler 2016 \\
\hline \multicolumn{4}{|l|}{ Metodología } \\
\hline Diseño & Retrospectivo & Retrospectivo & Prospectivo \\
\hline Tipo & Prueba diagnóstica & Prueba diagnóstica & Prueba diagnóstica \\
\hline \multicolumn{4}{|l|}{ Participantes } \\
\hline País & Taiwán & Puerto Rico & Cambodia \\
\hline Edad & $<18$ años & 5 a 15 años & 1 a 15 años \\
\hline $\mathrm{n}$ & 317 & 341 & 701 \\
\hline \# casos confirmados & 205 & 38 & 558 \\
\hline $\begin{array}{l}\text { Criterios de } \\
\text { inclusión }\end{array}$ & $\begin{array}{c}\text { Casos clínicos sospecho- } \\
\text { sos entre junio y diciem- } \\
\text { bre de } 2014\end{array}$ & $\begin{array}{c}\text { Casos sospechosos noti- } \\
\text { ficados al sistema de vigi- } \\
\text { lancia entre junio de } 2005 \\
\text { y mayo de } 2006\end{array}$ & $\begin{array}{l}\text { Casos clínicos sospecho- } \\
\text { sos entre septiembre de } \\
2011 \text { y enero de } 2013\end{array}$ \\
\hline Prueba índice & $\begin{array}{c}\text { Fiebre }\left(\mathrm{T} \geq 38^{\circ} \mathrm{C}\right) \text { y dos o } \\
\text { más: } \\
\text { Anorexia, } \\
\text { náuseas, } \\
\text { vómito, } \\
\text { rash, } \\
\text { cefalea, } \\
\text { dolor óseo, } \\
\text { mialgia, manifestaciones } \\
\text { hemorrágicas, leucope- } \\
\text { nia, trombocitopenia, sos- } \\
\text { pecha clínica de dengue }\end{array}$ & $\begin{array}{c}\text { Fiebre }\left(\mathrm{T} \geq 38^{\circ} \mathrm{C}\right) \text { y dos o } \\
\text { más: } \\
\text { Cefalea, } \\
\text { rash, } \\
\text { dolor ocular, } \\
\text { mialgia, } \\
\text { artralgia, hipotensión, } \\
\text { hemorragia, hemoconcen- } \\
\text { tración, trombocitopenia, } \\
\text { sospecha clínica de dengue }\end{array}$ & $\begin{array}{c}\text { Fiebre }\left(\mathrm{T}:>37.5^{\circ} \mathrm{C}\right) \text { más } \\
\text { cualquiera de los signos } \\
\text { de alarma: Distención } \\
\text { abdominal, } \\
\text { vómito persistente, } \\
\text { edema, } \\
\text { sangrado de mucosas, } \\
\text { letargia/irritabilidad, hepa- } \\
\text { tomegalia }>2 \mathrm{~cm}\end{array}$ \\
\hline Patrón de referencia & $\begin{array}{c}\text { RT-PCR } \\
+ \text { NS1 } \\
+ \text { IgM o IgG }\end{array}$ & $\begin{array}{l}\text { Aislamiento viral } \\
\text { RT-PCR } \\
\text { o NS1 } \\
\text { o IgM }\end{array}$ & $\begin{array}{l}\text { Uno o más: } \\
\text { Aislamiento Viral, } \\
\text { RT-PCR, } \\
\text { IgM, } \\
\text { NS1 }\end{array}$ \\
\hline \multicolumn{4}{|l|}{ Diagnóstico } \\
\hline $\begin{array}{l}\text { Laboratorio positivo } \\
\text { para dengue }\end{array}$ & Dengue & Dengue & $\begin{array}{c}\text { Dengue con signos de } \\
\text { alarma }\end{array}$ \\
\hline Serotipo & 1 & 2 & $1,2,3,4$ \\
\hline $\begin{array}{l}\text { Laboratorio negativos } \\
\text { para dengue }\end{array}$ & $\begin{array}{c}\text { Influenza, parainfluenza, } \\
\text { virus sincitial respira- } \\
\text { torio, } \\
\text { adenovirus, enterovirus, } \\
\text { rotavirus }\end{array}$ & No especifican. & $\begin{array}{l}\text { Encefalitis viral japonesa } \\
\text { Otras enfermedades fe- } \\
\text { briles sin especificación. }\end{array}$ \\
\hline
\end{tabular}

n: Número de personas incluidos como muestra. T:Temperatura corporal. ${ }^{\circ} \mathrm{C}:$ Grados Celsius. PCR-RT: $\quad$ Reacción en Cadena de la Polimerasa en Tiempo Real. NS1: Proteína no estructural NS1 del virus del Dengue. IgM:Inmunoglobulina tipo M. IgG: Inmunoglobulina tipo G. cm:Centímetros 
Tabla 2 Resultados de relación entre prueba índice y patrón de referencia.

\begin{tabular}{|c|c|c|c|c|c|c|c|c|c|c|c|c|}
\hline \multirow{3}{*}{ Estudio } & \multirow{3}{*}{ Prueba índice } & \multicolumn{11}{|c|}{ Resultados } \\
\hline & & \multicolumn{3}{|c|}{ Con dengue } & \multicolumn{3}{|c|}{ Sin dengue } & \multirow{2}{*}{$\mathrm{N}$} & \multicolumn{4}{|c|}{$\%$} \\
\hline & & VP & FN & $\mathrm{n}$ & FP & VN & $\mathrm{n}$ & & Se & $\mathrm{Sp}$ & VPP & VPN \\
\hline \multirow{24}{*}{$\begin{array}{l}\text { Chen } \\
2018\end{array}$} & Cefalea & 66 & 139 & 205 & 37 & 75 & 112 & \multirow{24}{*}{317} & 32.2 & 67 & 64.1 & 35 \\
\hline & Dolor retro-ocular & 13 & 192 & 205 & 15 & 97 & 112 & & 6.3 & 86.6 & 46.4 & 33.6 \\
\hline & Dolor óseo & 11 & 194 & 205 & 6 & 106 & 112 & & 5.4 & 94.6 & 64.7 & 35.3 \\
\hline & Mialgia & 66 & 139 & 205 & 46 & 66 & 112 & & 32.2 & 58.9 & 58.9 & 32.2 \\
\hline & Dolor abdominal & 50 & 155 & 205 & 20 & 92 & 112 & & 24.4 & 82.1 & 71.4 & 37.2 \\
\hline & Nausea/vómito & 67 & 138 & 205 & 33 & 79 & 112 & & 32.7 & 70.5 & 67.0 & 36.4 \\
\hline & Diarrea & 27 & 178 & 205 & 22 & 90 & 112 & & 13.2 & 80.4 & 55.1 & 33.6 \\
\hline & Tos & 54 & 151 & 205 & 60 & 52 & 112 & & 26.3 & 46.4 & 47.4 & 25.6 \\
\hline & Dolor faríngeo & 19 & 186 & 205 & 21 & 91 & 112 & & 9.3 & 81.3 & 47.5 & 32.9 \\
\hline & Rinorrea & 43 & 162 & 205 & 42 & 70 & 112 & & 21 & 62.5 & 50.6 & 30.2 \\
\hline & Rash & 156 & 49 & 205 & 50 & 62 & 112 & & 76.1 & 55.4 & 75.7 & 55.9 \\
\hline & Prurito & 59 & 146 & 205 & 18 & 94 & 112 & & 28.8 & 83.9 & 76.6 & 39.2 \\
\hline & Petequia & 30 & 175 & 205 & 7 & 105 & 112 & & 14.6 & 93.8 & 81.1 & 37.5 \\
\hline & Rash + Cefalea & 54 & 151 & 205 & 11 & 101 & 112 & & 26.3 & 90.2 & 83.1 & 40.1 \\
\hline & Petequias + Cefalea & 10 & 195 & 205 & 2 & 110 & 112 & & 4.9 & 98.2 & 83.3 & 36.1 \\
\hline & Rash + prurito + Petequias & 13 & 13 & 205 & 0 & 112 & 112 & & 6.3 & 100 & 100 & 36.8 \\
\hline & Duración fiebre & \multicolumn{2}{|c|}{$5(2-7 d)$} & 205 & \multicolumn{2}{|c|}{$4(1-15 d)$} & 112 & & & & & \\
\hline & Leucopenia & & & & & & & & $90(2-3)$ & $80(6)$ & $100(6)$ & \\
\hline & Trombocitopenia & & & & & & & & $81(2-7)$ & $92.9(6)$ & & \\
\hline & 囚TTPa & & & & & & & & & $50(0-7)$ & $80(1-4)$ & \\
\hline & 凶Transaminasas & & & & & & & & $68(5-7)$ & & $86(4-8)$ & \\
\hline & बPCR & & & & & & & & $89(2-9)$ & & $82(1-3)$ & \\
\hline & Leucopenia+Trombocitopenia & & & & & & & & & & $80(2-8)$ & \\
\hline & $\begin{array}{c}\text { Leucopenia + Trombocitopenia + } \\
\text { 『Transaminasas }\end{array}$ & & & & & & & & & & $00(2.6-8$ & \\
\hline \multirow{14}{*}{$\begin{array}{c}\text { Ramos } \\
2009\end{array}$} & Cefalea & 29 & 8 & 37 & 85 & 15 & 100 & \multirow{14}{*}{341} & 78.4 & 15.0 & 25.4 & 65.2 \\
\hline & Mialgia & 17 & 17 & 34 & 53 & 39 & 92 & & 50.0 & 42.4 & 24.3 & 69.6 \\
\hline & Rash & 14 & 20 & 34 & 4 & 84 & 88 & & 41.2 & 95.5 & 77.8 & 80.8 \\
\hline & Dolor ocular & 13 & 23 & 36 & 33 & 57 & 90 & & 36.1 & 63.3 & 28.3 & 71.3 \\
\hline & Artralgia & 9 & 27 & 36 & 28 & 68 & 96 & & 25.0 & 70.8 & 24.3 & 71.6 \\
\hline & Hemorragia & 3 & 28 & 31 & 4 & 92 & 96 & & 9.7 & 95.8 & 42.9 & 76.7 \\
\hline & Nausea + vómito & 10 & 21 & 31 & 26 & 70 & 96 & & 32.3 & 72.9 & 27.8 & 76.9 \\
\hline & Diarrea & 4 & 27 & 31 & 7 & 83 & 90 & & 12.9 & 92.2 & 36.4 & 75.5 \\
\hline & $\begin{array}{c}\text { Cualquier síntoma respiratorio } \\
\text { alto }\end{array}$ & 19 & 13 & 32 & 89 & 18 & 107 & & 59.4 & 16.8 & 17.6 & 58.1 \\
\hline & Dolor faríngeo & 16 & 15 & 31 & 68 & 32 & 100 & & 51.6 & 32.0 & 19.0 & 68.1 \\
\hline & Tos & 7 & 24 & 31 & 52 & 46 & 98 & & 22.6 & 46.9 & 11.9 & 65.7 \\
\hline & Rash + no dolor faríngeo & NP & $\mathrm{NP}$ & NP & NP & NP & NP & & 21.9 & 97.7 & 77.8 & 76.9 \\
\hline & Rash+ no congestión nasal & $\mathrm{NP}$ & $\mathrm{NP}$ & $\mathrm{NP}$ & NP & $\mathrm{NP}$ & $\mathrm{NP}$ & & 33.3 & 98.8 & 91.7 & 79.1 \\
\hline & Rash + no tos & $\mathrm{NP}$ & $\mathrm{NP}$ & $\mathrm{NP}$ & NP & NP & NP & & 37.9 & 100 & 100 & 82.4 \\
\hline \multirow{9}{*}{$\begin{array}{c}\text { Cavailler } \\
2016\end{array}$} & Rash & 155 & 403 & 558 & 25 & 118 & 143 & \multirow{9}{*}{701} & 27.8 & 82.5 & 86.1 & 22.7 \\
\hline & Dolor retro ocular & 160 & 398 & 558 & 23 & 120 & 143 & & 28.7 & 83.9 & 87.4 & 23.2 \\
\hline & Mialgia & 209 & 349 & 558 & 32 & 111 & 143 & & 37.5 & 77.6 & 86.7 & 24.1 \\
\hline & Mialgia/artralgia & 236 & 322 & 558 & 44 & 99 & 143 & & 42.3 & 69 & 84.2 & 23.5 \\
\hline & anorexia & 351 & 207 & 558 & 77 & 66 & 143 & & 62.9 & 46.2 & 82.0 & 24.2 \\
\hline & Vomito & 440 & 118 & 558 & 90 & 53 & 143 & & 78.9 & 37.1 & 83.0 & 31.1 \\
\hline & Nausea o vomito & 459 & 99 & 558 & 99 & 50 & 143 & & 82.3 & 30.8 & 82.3 & 30.8 \\
\hline & Dolor abdominal & 476 & 82 & 558 & 106 & 37 & 143 & & 85.3 & 25.9 & 81.8 & 31.1 \\
\hline & Letargia / irritabilidad & 317 & 241 & 558 & 69 & 74 & 143 & & 56.8 & 51.4 & 82.0 & 23.4 \\
\hline
\end{tabular}

En rojo los valores calculados para aquellos signos y síntomas que contaban con datos suficientes para tal fin. N: número de sujetos analizados VP: Verdaderos Positivos; FN: Falsos Negativos; FP: Falsos Positivos; VN: Verdaderos Negativos; Se: Sensibilidad; Sp: Especificidad; VPP: Valor Predictivo Positivo; VPN: Valor Predictivo Negativo 
Gráfico 2. Evaluación de la calidad QUADAS-2: resumen

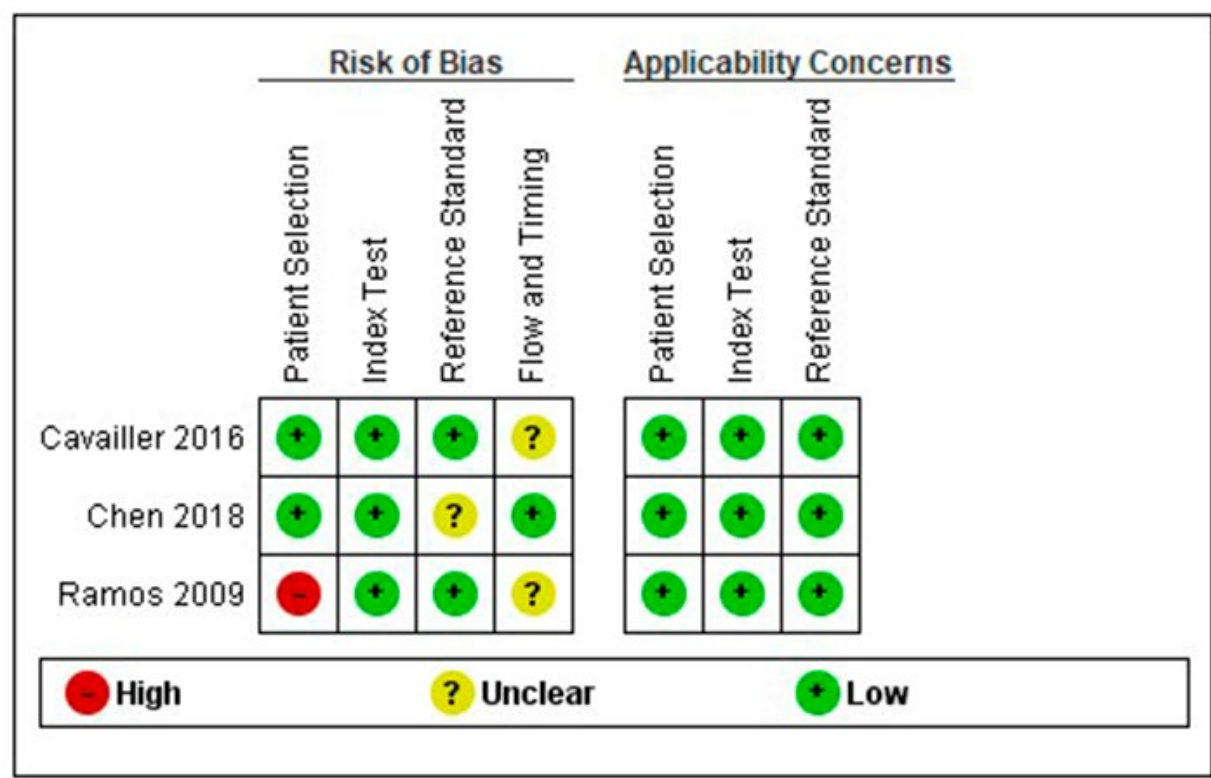

Fuente: elaboración propia en software RevMan V. 5.3

Gráfico 3. Evaluación de la calidad QUADAS-2. Porcentajes por dominio evaluado

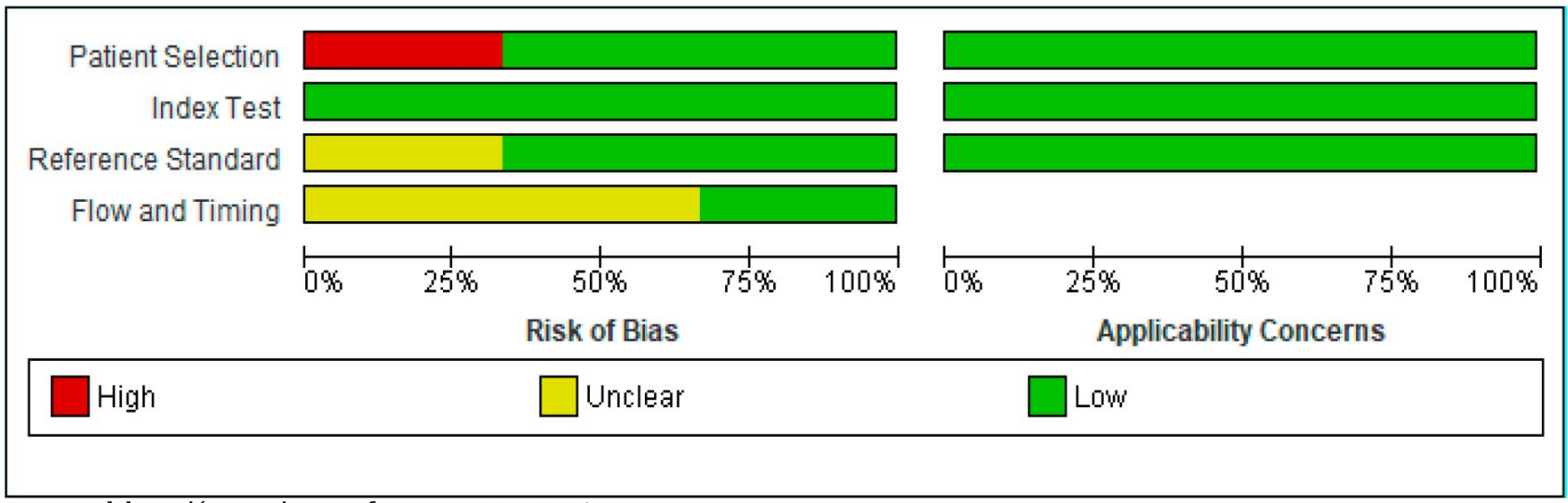

Fuente: elaboración propia en software RevMan V. 5.3

sospeche la enfermedad del dengue, teniendo en cuenta que presentan espeficidad y valor predictivo positivo del $100 \%$, aun cuando la sensibilidad es muy baja (tabla 2). Así, se resalta la importancia de su presencia o ausencia en un individuo pediátrico en quien se sospeche una infección por el virus del dengue.-

En cuanto a la confirmación diagnóstica, múltiples autores de estudios de pruebas diagnósticas, como el CDC (De las siglas en inglés de: Centers for disease Control) en 2017 (24), han logrado establecer la mejora en la capacidad diagnostica para la infección por el virus del dengue mediante la combinación de las diferentes técnica de laboratorio, la PCR-RT para dengue, la prueba de NS1, la Inmunoglobulina tipo $\mathrm{M}$ o el aumento en las diluciones de la Inmunoglobulina tipo $\mathrm{G}$ y el aislamiento viral, pudiéndose establecer como método confirmatorio cualquier combinación. Sin embargo, la combinación empleada en cada estudio fue diferente, utilizando como patrón de oro una o más pruebas de referencia. Mientras que Chen y cols. evaluaron contra una combinación de pruebas [PCR-RT + NS1 + IgM o IgG], Ramos y Cavailler realizaron la confirmación diagnóstica con una o más de las pruebas disponibles. Esto afecta las características operativas de cada patrón de oro configurado, impidiendo así la comparación de la validez diagnóstica de los signos y síntomas clínicos evaluados para el diagnóstico diferencial de la infección por el virus del dengue.

De otra parte, los estudios realizados por Hammond y cols. en el 2005 (25) y Nunes y cols. en el 2016 (26) indican que la presentación clínica de la enfermedad causada por el virus del dengue cambia de acuerdo con la edad de la persona infectada, siendo pertinentes los estudios que evalúan las características operativas de los signos y síntomas en la población pediátrica con dengue. Sin embargo, la heterogeneidad metodológica y 
de población entre los artículos incluidos en esta revisión impiden el metaanálisis de la información, haciendo imposible resumir los criterios de diagnóstico diferencial utilizados para la identificación clínica de la infección por el virus del dengue en la población pediátrica.

Aunque durante la atención medica rutinaria de la población pediátrica con sospecha de dengue está indicada la solicitud del hemograma, tal como lo establecen las guías internacionales de práctica clínica OMS 2009 (3) y OMS 2016 (7), tan solo uno de los estudios incluidos en esta revisión, la de Chen y cols. tiene en cuenta los hallazgos de este paraclínico para procesar la información, siendo evidente la necesidad de conocer más, respecto a los hallazgos en el hemograma y su valor diagnóstico para el paciente pediátrico con dengue.

Las limitaciones de la presente revisión se resumen en la abundante literatura gris y de estudios descriptivos sobre el diagnóstico de dengue y los brotes que se presentan periódicamente, con pocos estudios de prueba diagnóstica que permitan la comparación de síntomas y signos con pruebas diagnósticas estandarizadas. Aunque se efectuó una búsqueda exhaustiva de estudios, es posible que queden por fuera algunos estudios del conocimiento de los expertos temáticos que pudiesen alimentar la revisión, pero no hay antecedentes de eventos que incluyan un panel de expertos para profundizar al respecto, por lo cual se considera que la información específica sobre el tema es escasa. A través de esta revisión sistemática, no se encontró la evidencia suficiente y homogénea que permita realizar un metaanálisis de la información para establecer las características operativas de los hallazgos al examen físico de la población pediátrica con dengue, evidenciando así una necesidad.

Finalmente, teniendo en cuenta el trabajo realizado y expuesto en el presente documento, se sugiere el desarrollo de estudios de prueba diagnóstica con los cuales se llene este vacío del conocimiento y se mejore la precisión diagnóstica y consecuente tratamiento de los pacientes pediátricos con la enfermedad causada por el virus del dengue, además de generar evidencia que permita contar con opciones como modelos predictivos para diagnóstico clínico diferencial del dengue en pediatría.

\section{Declaración de conflictos de intereses}

Los autores declaramos no tener ningún conflicto de interes.

\section{R E F E R E N C I A S}

1. Cumakov MP, Doherty RL, Downs WG, Hammon MC, Panthier $\mathrm{R}$, Porterfield JS, et al. Los arbovirus y su importancia en patología humana. 1967.p. 1-86.

2. Rodriguez-Morales AJ, Villamil-Gómez WE, Franco-Paredes C. The arboviral burden of disease caused by co-circulation and co-infection of dengue, chikungunya and Zika in the Americas. Travel Med Infect Dis. 2016;14(3):177-9.

3. Organización Panamericana de la Salud. Organización Mundial de la Salud Dengue: Guías para el diagnóstico, tratamiento, prevención y control. La Paz. Bolivia. OPS/OMS 2009: 113-5.
4. San Martín JL, Brathwaite-Dick O. La estrategia de gestión integrada para la prevención y el control del dengue en la región de Las Américas. Rev Panam Salud Pública. 2017;21(1):5563.

5. Oficina Regional para Las Américas. OPS OMS | Descripción de la situación epidemiológica actual del dengue en las Américas. [consultado en Julio 31 del 2018]. Disponible en: https://www. paho.org/hq/index.php?option=com_content\&view =article\&id $=4494 \&$ Itemid $=2481 \&$ lang $=e s$

6. Mayer S V., Tesh RB, Vasilakis N. The emergence of arthropodborne viral diseases: A global prospective on dengue, chikungunya and zika fevers. Acta Trop. 2017;166:155-63.

7. Organización Panamericana de la Salud. Dengue: guías para la atención de enfermos en la Región de Las Américas. 2 ed. Washington, DC, 2016. 123p. Disponible en https://iris.paho. org/bitstream/handle/10665.2/28232/9789275318904_esp. pdf? sequence $=1$ \&isAllowed $=y$

8. Instituto Nacional de Salud. Protocolo de Vigilancia en Salud Pública Dengue Versión 4. Bogotá, Colombia, 29 de diciembre de 2017. Disponible: https://www.ins.gov.co/Noticias/ Dengue/7.\%20Dengue\%20PROTOCOLO.pdf

9. Ministerio de Salud y Protección Social, Instituto Nacional de Salud, OPS/OMS. Guía de atención clínica integral del paciente con dengue. Bogotá, Colombia, 2010. Disponible en: http:// manizalessalud.net/wp content/uploads/2019/03/guia_para_ atencion.pdf

10. Ministerio de Salud y Protección Social. Lineamientos para la atención clínica integral de pacientes con zika en Colombia. Bogotá, marzo de 2016. Disponible en: https://www.minsalud. gov.co/sites/rid/Lists/Bib liotecaDigital/RIDE/VS/PP/ET/ lineamientos-atencion-clinica-integral-paciente-zikacolombia.pdf

11. Martínez-Vega RA, Díaz-Quijano FA, Villar-Centeno LA. Dificultad para el diagnóstico clínico temprano del dengue en un área endémica y su impacto sobre el manejo médico inicial. Rev Med Chile 2006;134(9):1153-60.

12. BhatVG, Chavan P, Ojha S, Nair PK. Challenges in the Laboratory Diagnosis and Management of Dengue Infections. Open Microbiol J. 2015;31;9:33-7.

13. Guzmán MG, Gubler DJ, Izquierdo A, Martínez E, Halstead SB. Dengue infection Nat Rev Dis Primers. 2016;18;2:16055.

14. Kantor IN. Dengue, zika y chikungunya. Medicina (Buenos Aires). 2016;76(2):93-7. Epub 2016 Feb 22.

15. Paniz-Mondolfi AE, Rodríguez-Morales AJ, Blohm G, Márquez M, Villamil-Gómez WE. ChikDenMaZika Syndrome: the challenge of diagnosing arboviral infections in the midst of concurrent epidemics. Ann Clin Microbiol Antimicrob. 2016;15(1):42.

16. Moher D, Liberati A, Tetzlaff J, Altman DG, Altman D, Antes G, et al. Preferred reporting items for systematic reviews and meta-analyses: The PRISMA statement (Chinese edition). J Chinese Integr Med. 2009;7(9):889-96.

17. Whiting PF1, Rutjes AW, Westwood ME, Mallett S, Deeks JJ, Reitsma JB, et al. QUADAS-2: a revised tool for the quality assessment of diagnostic accuracy studies. Ann Intern Med. 2011;18;155(8):529-36.

18. Chen CH, Huang YC, Kuo KC, Li CC. Clinical features and dynamic ordinary laboratory tests differentiating dengue fever from other febrile illnesses in children. J Microbiol Immunol Infect. 2018;51(5):614-620.

19. Ramos MM, Tomashek KM, Arguello DF, Luxemburger C, Quiñones L, Lang J, et al. Early clinical features of dengue infection in Puerto Rico. Trans $\mathrm{R}$ Soc Trop Med Hyg. 2009;103(9):878-84. 
20. Cavailler P, Tarantola A, Leo YS, Lover AA, Rachline A, Duch M, et al. Early diagnosis of dengue disease severity in a resourcelimited Asian country. BMC Infect Dis. 2016;16(1):512.

21. Bhattacharya MK, Maitra S, Ganguly A, Bhattacharya A, Sinha A. Dengue: a growing menace -- a snapshot of recent facts, figures \& remedies. Int J Biomed Sci. 2013 Jun;9(2):61-7.

22. Guo C, Zhou Z, Wen Z, Liu Y, Zeng C, Xiao D, et al. Global Epidemiology of Dengue Outbreaks in 1990-2015: A Systematic Review and Meta-Analysis. Front Cell Infect Microbiol. 2017 Jul 12;7:317.

23. Messina JP, Brady OJ, Golding N, Kraemer MUG, Wint GRW, Ray SE, et al. The current and future global distribution and population at risk of dengue. Nat Microbiol. 2019 Jun 10. doi: 10.1038/s41564-019-0476-8. [Epub ahead of print]

24. Centers for Disease Control and Prevention (CDC). Prueba Trioplex RCP-TR en tiempo real. Indicaciones para su uso. 2017. https://www.cdc.gov/zika/pdfs/trioplex-real-time-rt-pcrassay-instructions-for-use.pdf

25. Hammond SN, Balmaseda A, Pérez L, Téllez Y, Saborío SI, Mercado JC, et al. Differences in dengue severity in infants, children, and adults in a 3-year hospital-based study in Nicaragua. Am J Trop Med Hyg. 2005;73(6):1063-704

26. Nunes PC, Sampaio SA, da Costa NR, de Mendonça MC, Lima Mda R, Araujo SE, et al. Dengue severity associated with age and a new lineage of dengue virus-type 2 during an outbreak in Rio De Janeiro, Brazil. J Med Virol. 2016;88(7):1130-6. 